\title{
Betweenness in order-theoretic trees
}

\author{
Bruno COURCELLE \\ LaBRI, CNRS, \\ 351 Cours de la Libération, \\ 33405 Talence, France \\ courcell@labri.fr
}

\begin{abstract}
The ternary betweenness relation of a tree, $B(x, y, z)$, indicates that $y$ is on the unique path between $x$ and $z$. This notion can be extended to order-theoretic trees defined as partial orders such that the set of nodes greater than any node is linearly ordered. In such generalized trees, the unique "path" between two nodes can have infinitely many nodes.

We generalize some results obtained in a previous article for the betweenness of join-trees. Join-trees are order-theoretic trees such that any two nodes have a least upper-bound. The motivation was to define conveniently the rank-width of a countable graph. We have called quasitree the betweenness relation of a join-tree. We proved that quasi-trees are axiomatized by a first-order sentence.

Here, we obtain a monadic second-order axiomatization of betweenness in order-theoretic trees. We also define and compare several induced betweenness relations, i.e., restrictions to sets of nodes of the betweenness relations in generalized trees of different kinds. We prove that induced betweenness in quasi-trees is characterized by a first-order sentence. The proof uses order-theoretic trees.

Keywords : Betweenness, order-theoretic tree, join-tree, first-order logic, monadic second-order logic, quasi-tree.
\end{abstract}

\section{Introduction}

In order to define the rank-width of a countable graph in such a way that it be the least upper-bound of those of its finite induced subgraphs, we defined in [3] generalized undirected trees called quasi-trees such that the unique "path" (in a precise sense extending the usual notion) between any two nodes can have infinitely many nodes, in particular, can have the order-type of the interval $[0,1]$ of rational numbers. A related notion is that of an order-theoretic tree defined as a partial order such that the set of nodes greater than any node is linearly ordered. It is a join-tree if any two nodes have a least upper-bound. It may have no root, i.e., no largest element. Quasi-trees can be seen as undirected join-trees. 
The betweenness relation of a usual tree is the ternary relation $B$, such that $B(x, y, z)$ holds if and only if $x, y, z$ are distinct and $y$ is on the unique path between $x$ and $z$. This notion can be generalized to order-theoretic trees. A quasi-tree is the betweenness relation of a countable (which means possibly finite) join-tree, and quasi-trees are the countable structures $(N, B)$ that satisfy (hence, are axiomatized by) a first-order sentence. We also obtained in [2,3] an algebraic characterization of the join-trees and quasi-trees that are the unique countable models of monadic-second order sentences. This type of characterization will be extended to order-theoretic trees in a future work. In this article, we obtain a monadic second-order axiomatization for betweenness in order-theoretic trees.

We also define and study several induced betweenness relations, i.e., the restrictions to sets of nodes of betweenness in generalized trees of different kinds. An induced betweenness in a quasi-tree need not be a quasi-tree. However, induced betweenness in quasi-trees is also characterized by a single first-order sentence, which does not follow immediately from the first-order characterization of quasi-trees by a general logical argument. The proof uses order-theoretic trees.

We obtain four types of betweenness and induced betweenness relations $S=$ $(N, B)$. In each case, such a structure $S$ is defined from an order-theoretic tree $T$. Except for the case of induced betweenness in order-theoretic trees, some defining tree $T$ can be described in $S$ by monadic second-order formulas. In technical words, $T$ is defined from $S$ by a monadic second-order transduction (see [6] for a thorough study).

In order to obtain a concrete view of our generalized trees, we embed them in topological trees, defined as connected unions of segments of straight lines in the plane that have no subset homeomorphic to a circle. Induced betweenness relations in topological trees and in quasi-trees are the same.

Other works on betweenness.

Betweenness in partial orders of any cardinality is axiomatized by J. Lihova in [7] by an infinite set of universal first-order sentences. It is not stated whether this set can be replaced by a finite one, but presumably not. It can be by a monadic second-order sentence ${ }^{1}$.

Motivated by the study of convex geometries, V. Chvatal studies in [1] betweenness in finite triangulated graphs. It is relative to induced paths : $y$ is between $x$ and $z$ if it is an intermediate vertex on a chordless path between $x$ and $z$. No axiomatization is provided.

Complete proofs for all stated results and counter-examples can be found in [5], which can be read on line at : https://hal.archives-ouvertes.fr/hal-02205829.

\section{Definitions and basic facts}

All sets, trees, graphs and logical structures are countable, which means, finite or countably infinite. If $n$ is a positive integer, then $[n]:=\{1,2, \ldots, n\}$.

\footnotetext{
1 Betweenness in partial orders, work in preparation.
} 


\subsection{Trees}

A tree is a possibly empty, undirected graph that is connected and has no cycles. Hence, it has no loops and no two edges with same end vertices. The set of nodes of a tree $T$ is $N_{T}$.

A rooted tree is a nonempty tree equipped with a distinguished node called its root. We define on $N_{T}$ the partial order $\leq_{T}$ such that $x \leq_{T} y$ if and only if $y$ is on the unique path between $x$ and the root. The least upper-bound of $x$ and $y$, denoted by $x \sqcup_{T} y$ is their least common ancestor, also called their join. The minimal elements are the leaves, and the root is the greatest node.

Fact 1 : A partial order $(N, \leq)$ is $\left(N_{T}, \leq_{T}\right)$ for some rooted tree $T$ if and only if it has a largest element and, for each $x \in N$, the set $L_{\geq}(x):=\{y \in N \mid x \leq y\}$ is finite and linearly ordered. These conditions imply that any two nodes have a join.

\subsection{Order-theoretic forests and trees}

Definition 2 : O-forests and $O$-trees.

In order to have a simple terminology, we will use the prefix O- to mean order-theoretic and to distinguish these generalized trees from those of [4].

(a) An $O$-forest is a pair $F=(N, \leq)$ such that:

1) $N$ is a possibly empty set called the set of nodes,

$2) \leq$ is a partial order on $N$ such that, for every node $x$, the set $L_{\geq}(x)$ is linearly ordered.

It is an $O$-tree if furthermore:

3 ) every two nodes $x$ and $y$ have an upper-bound.

An O-forest is thus the disjoint union of O-trees that are its connected components, with respect to its Gaifman graph ${ }^{2}$. Two nodes are in a same composing O-tree if and only if they have an upper-bound.

The leaves are the minimal elements. If $N$ has a largest element $r(x \leq r$ for all $x \in N$ ) then $F$ is a rooted $\mathrm{O}$-tree and $r$ is its root.

(b) An O-tree $T$ is a join-tree $e^{3}$ if every two nodes $x$ and $y$ have a least upper-bound denoted by $x \sqcup_{T} y$ and called their join (cf. Subsection 1.1).

\section{Examples and remarks 3 :}

(1) If $T$ is a rooted tree, then $\left(N_{T}, \leq_{T}\right)$ is a join-tree. Every finite O-tree is a join-tree of this form.

(2) Every linear order is (trivially) a join-tree.

(3) Let $S:=\mathbb{N} \cup\{a, b, c\}$ be strictly ordered by $<_{S}$ such that $a<_{S} b, c<_{S} b$ and $b<_{S} i<_{S} j$ for all $i, j \in \mathbb{N}$ such that $j<i$, and $a$ and $c$ are incomparable. Then $\left(S, \leq_{S}\right)$ is a join-tree. In particular $a \sqcup_{S} c=b$. It is not the partial order

${ }^{2}$ Defined for a relational structure: two elements are adjacent if they belong to some tuple of some relation.

${ }^{3}$ An ordered tree is a rooted tree such that the set of sons of any node is linearly ordered. This notion is extended in [4] to join-trees. Ordered join-trees should not be confused with order-theoretic trees, that we call O-trees for simplicity. 
associated with any rooted tree by Fact 1 . If $S^{\prime}:=S-\{b\}$, we obtain an O-tree with set of nodes $S^{\prime}$. It is not a join-tree because $a$ and $c$ have no join.

(4) We can consider $\mathbb{N} \cup\{a, b\}$ as forming a path ${ }^{4}$ in the join-tree $\left(S, \leq_{S}\right.$ ) (of (3)) between $a$ and 0 , the largest element.

\section{Quasi-trees and betweenness in O-trees}

In this section, we will define a betweenness relation in O-trees, and compare it with the betweenness relation induced by sets of nodes of join-trees or O-trees. We generalize the notion of quasi-tree defined and studied in [3] and [4].

For a ternary relation $B$ on a set $N$ and $x, y \in N$, we define $[x, y]_{B}:=$ $\{x, y\} \cup\{z \in N \mid(x, z, y) \in B\}$. If $n>2$, then the notation $\neq\left(x_{1}, x_{2}, \ldots, x_{n}\right)$ means that $x_{1}, x_{2}, \ldots, x_{n}$ are pairwise distinct.

\subsection{Betweenness in trees and quasi-trees}

Definition $4:$ Betweenness in linear orders and trees.

(a) Let $L=(X, \leq)$ be a linear order ${ }^{5}$. Its betweenness relation $B_{L}$ is the ternary relation on $X$ defined by :

$B_{L}(x, y, z): \Longleftrightarrow x<y<z$ or $z<y<x$.

(b) If $T$ is a tree or a forest, its betweenness relation $B_{T}$ is the ternary relation on $N_{T}$ defined by :

$B_{T}(x, y, z): \Longleftrightarrow x, y, z$ are pairwise distinct and $y$ is on the unique path between $x$ and $z$.

If $R$ is a rooted tree, we define its betweenness relation $B_{R}$ as $B_{U n d(R)}$ where $\operatorname{Und}(R)$ is the tree obtained from $R$ by forgetting its root and its edge directions. We have :

$B_{R}(x, y, z) \Longleftrightarrow x, y, z$ are pairwise distinct, $x$ and $z$ have a join $x \sqcup_{R} z$ and $\left(x<_{R} y \leq_{R} x \sqcup_{R} z\right.$ or $\left.z<_{R} y \leq_{R} x \sqcup_{R} z\right)$.

(c) With a ternary relation $B$ on a set $X$, we associate the ternary relation $A$, also on $X$ :

$$
A(x, y, z): \Longleftrightarrow B(x, y, z) \vee B(x, z, y) \vee B(y, x, z) .
$$

\footnotetext{
${ }^{4}$ Formal definition in [5].

${ }^{5}$ This definition can be used in partial orders. The corresponding notion of betweenness is axiomatized in [7]. We will not use it for defining betweenness in ordertheoretic trees, although these trees are defined as partial orders.
} 
It is to be read $: x, y, z$ are aligned.

If $n \geq 3$, then $B^{+}\left(x_{1}, x_{2}, \ldots, x_{n}\right)$ stands for the conjunction of the conditions $B\left(x_{i}, x_{j}, x_{k}\right)$ for all $1 \leq i<j<k \leq n$ and all $1 \leq k<j<i \leq n$.

Proposition 5 [Proposition 5.2 in [4]] : (a) The betweenness relation $B$ of a linear order $(X, \leq)$ satisfies the following properties for all $x, y, z, u \in X$.

$$
\begin{aligned}
& \mathrm{A} 1: B(x, y, z) \Rightarrow \neq(x, y, z) \text {. } \\
& \mathrm{A} 2: B(x, y, z) \Rightarrow B(z, y, x) \text {. } \\
& \text { A3 }: B(x, y, z) \Rightarrow \neg B(x, z, y) \text {. } \\
& \mathrm{A} 4: B(x, y, z) \wedge B(y, z, u) \Rightarrow B(x, y, u) \wedge B(x, z, u) \text {. } \\
& \mathrm{A} 5: B(x, y, z) \wedge B(x, u, y) \Rightarrow B(x, u, z) \wedge B(u, y, z) \text {. } \\
& \mathrm{A} 6: B(x, y, z) \wedge B(x, u, z) \Rightarrow y=u \vee[B(x, u, y) \wedge B(u, y, z)] \\
& \mathrm{A} 7^{\prime}: \neq(x, y, z) \Rightarrow A(x, y, z) \text {. } \\
& \vee[B(x, y, u) \wedge B(y, u, z)] .
\end{aligned}
$$

(b) The betweenness relation $B$ of a tree $T$ satisfies the properties A1-A6 for all $x, y, z, u$ in $N_{T}$ together with the following weakening of A7' :

$$
\mathrm{A} 7: \neq(x, y, z) \Rightarrow A(x, y, z) \vee \exists w \cdot(B(x, w, y) \wedge B(y, w, z) \wedge B(x, w, z)) .
$$

Remarks 6 : (1) A7' says that if $x, y, z$ are three elements in a linear order, then, one of them is between the two others. Property A7 says that, in a tree $T$, if $x, y, z$ are three nodes not on a path, then there is some node $w$ between any two of them. Actually :

$\{w\}=P_{x, y} \cap P_{y, z} \cap P_{x, z}$ where $P_{u, v}$ is the set of nodes on the path between $u$ and $v$,

so that we have $B(x, w, y) \wedge B(y, w, z) \wedge B(x, w, z)$.

If $T$ is a rooted tree, then $w$ is the least common ancestor of $x, y$ and $z$. In the tree $T$ of Figure 3(b) below, we have $w=1$ if $x=a, y=d$ and $z=e$.

(2) Properties A1-A6 imply that the two cases of the conclusion of A7 are exclusive $^{6}$ and that, in the second one, there is a unique node $w$ satisfying $B(x, w, y) \wedge B(y, w, z) \wedge B(x, w, z)$ (by Lemma 11 of [3]), that is denoted by $M_{S}(x, y, z)$.

(3) Properties A1-A5 belong to the axiomatization of betweenness in partial orders given in [7].

The letter $B$ and its variants, $B_{T}, B_{1}$, etc. will denote ternary relations.

Definitions 7 : More betweenness properties.

We define the following properties of a structure $(N, B)$ :

\footnotetext{
${ }_{6}$ The three cases of $A(x, y, z)$ are exclusive by $\mathrm{A} 2$ and $\mathrm{A} 3$.
} 


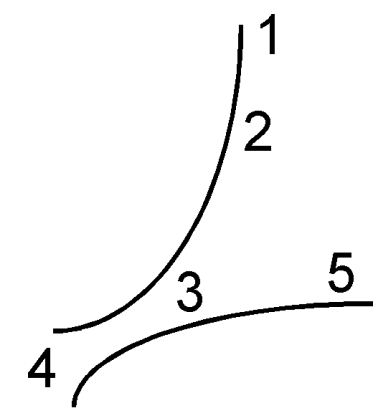

Fig. 1. Structure $S$ of Example 8 .

$$
\begin{gathered}
\mathrm{A} 8: \forall u, x, y, z \cdot[\neq(u, x, y, z) \wedge B(x, y, z) \Rightarrow \\
B(u, x, y) \vee B(u, y, z) \vee B(x, y, u) \vee B(y, z, u)] . \\
\mathrm{A} 8 ': \forall u, x, y, z \cdot[\neq(u, x, y, z) \wedge B(x, y, z) \wedge \neg A(y, z, u) \Rightarrow B(x, y, u)] .
\end{gathered}
$$

If $(N, B)$ satisfies $\mathrm{A} 1-\mathrm{A} 6$, the four cases of the conclusion of $\mathrm{A} 8$ are not exclusive : $B(u, x, y)$ implies $B(u, y, z)$ (because of $B(x, y, z)$ and $\mathrm{A} 4$ ).

Example 8 : A1-A6 do not imply A8'.

Consider $S:=([5], B)$ where $B$ satisfies (only) $B^{+}(1,2,3,4) \wedge B^{+}(5,3,4)$. It is shown in Figure 1. (There is no curve line going through $1,2,5$ because $B(1,2,5)$ is not assumed to be valid).

Conditions A1-A6 hold but A8' does not, because we have $B(1,2,3) \wedge \neg A(2,3,5)$ : A8' would imply $B(1,2,5)$ that is not assumed. By the next lemma, A1-A6 do not imply A8 either.

In the following proofs and discussions about a structure $(N, B)$, we will always assume (unless otherwise specified) that A1-A6 hold, and we will not make their use explicit. We say that $(N, B)$ is trivial if $B=\emptyset$. In this case, Properties A1-A6, A8 and A8' hold trivially.

Lemma 9 : Let $(N, B)$ satisfy A1-A6.

(1) A8 is equivalent to A8'.

(2) A7 implies A8, and thus, A8'.

(3) If A8 holds, then the Gaifman graph of $(N, B)$ is either edgeless (if $B=\emptyset$ ) or connected.

Definition 10 : Quasi-trees [3].

(a) A quasi-tree is a structure $S=(N, B)$ such that $B$ is a ternary relation on $N$, called the set of nodes, that satisfies conditions A1-A7. To avoid uninteresting special cases, we also require that $N$ has at least 3 nodes. We say that $S$ is discrete if $[x, y]_{B}:=\{z \mid B(x, z, y)\}$ is finite for all $x, y$.

(b) From a join-tree $J=(N, \leq)$, we define a ternary relation $B_{J}$ on $N$ by : 


$$
B_{J}(x, y, z): \Longleftrightarrow \neq(x, y, z) \wedge\left(\left[x<y \leq x \sqcup_{J} z\right] \vee\left[z<y \leq x \sqcup_{J} z\right]\right),
$$

called its betweenness relation. Here, we take as a definition, the characterization of $B_{R}$ for rooted trees given in Definition 4(b). Note that $x \sqcup_{J} z$ is always defined.

Theorem 11 [Proposition 5.6 of [4]] :

(1) The structure $q t(J):=\left(N, B_{J}\right)$ associated with a join-tree $J=(N, \leq)$ with at least 3 nodes is a quasi-tree. Every quasi-tree is $q t(J)$ for some join-tree $J$.

(2) A quasi-tree is discrete if and only if it is $q t(T)$ for some rooted tree $T$ (that is a join-tree defined as the partial order $\left(N_{T}, \leq_{T}\right)$ ).

In this article, we will rather think of quasi-trees as betweenness relations of join-trees, axiomatized by A1-A7.

\subsection{Other betweenness relations}

If $B$ is a ternary relation on $V$ and $X \subseteq V$, then $B[X]:=B \cap(X \times X \times X)$ is the induced relation of $B$ on $X$.

Definition 12 : Induced betweenness in a quasi-tree

If $Q=(N, B)$ is a quasi-tree, $X \subseteq N$, we say that $Q[X]:=(X, B[X])$ is an induced betweenness in $Q$. It is induced on $X$. It need not be a quasi-tree because A7 does not hold for a triple $(x, y, z)$ such that $M_{Q}(x, y, z)$ is not in $X$ (cf. Proposition 5 and Remarks 6).

We will prove that a ternary relation is an induced betweenness in a quasi-tree if and only if it satisfies Properties A1-A6 and A8. The proof uses O-trees.

Proposition 13 : An induced betweenness in a quasi-tree satisfies properties A1-A6 and A8.

Proof: The sentences expressing A1-A6 and A8 are universal.The validity of such sentences is preserved under taking induced substructures (we are dealing with relational structures). The result follows from Theorem 11 and Lemma 9(2) showing that a quasi-tree satisfies A8.

Definition 14 : Betweenness in O-forests.

(a) From an O-forest $F=(N, \leq)$, we define a ternary relation $B_{F}$ on $N$, called its betweenness relation, by :

$$
B_{F}(x, y, z): \Longleftrightarrow \neq(x, y, z) \wedge[(x<y \leq x \sqcup z) \vee(z<y \leq x \sqcup z)] .
$$

where the join $x \sqcup z$ must be defined.

(b) If $F=(N, \leq)$ is an O-forest and $X \subseteq N$, then $\left(X, B_{F}[X]\right)$ is an induced betweenness relation in $F$. 
Remark 15: The difference with Definition 10(b) is that if $x$ and $z$ have no least upper-bound, i.e., if $x \sqcup z$ is undefined, then $B_{F}$ contains no triple of the form $(x, y, z)$. If $F$ is a finite O-tree, it is a join-tree and thus, $\left(N, B_{F}\right)$ is a quasi-tree.

Thus we have four classes of betweenness relations $S=(N, B)$ : quasi-trees, induced betweenness in quasi-trees, betweenness and induced betweenness in O-forests.

Here are some easy observations.

(1) The induced betweenness $(X, B)$ on a set $X$ of leaves of a tree is trivial, which means that $B=\emptyset$.

(2) The Gaifman graph of a betweenness structure $S$ is connected in the following cases : $S$ is a quasi-tree, or it is a nontrivial induced betweenness in a quasi-tree (by A8) or it is the betweenness relation of an O-tree with at least 3 nodes (easy proof). It may be not connected in the other cases.

(3) If $S$ is an induced betweenness in an O-forest consisting of several disjoint O-trees, then two nodes in the different O-trees cannot belong to a same triple, and as a consequence, cannot be linked by a path in the Gaifman graph of $S$. Hence, a structure $(N, B)$ is the betweenness of an O-forest, or an induced betweenness in an O-forest if and only if each of its connected components is so in an O-tree. We will only consider betweenness of O-trees (class BO) and induced betweenness in O-trees (class IBO).

We will denote by QT the class of quasi-trees and by IBQT the class of induced betweenness relations in quasi-trees. Figure 2 illustrates the following inclusions.

Proposition 16 : We have the following strict inclusions :

$$
\text { QT } \subset \text { IBQT, } \mathbf{Q T} \subset \mathbf{B O} \subset \mathbf{I B O} \text { and } \mathbf{Q T} \subset \mathbf{I B Q T} \cap \mathbf{B O} .
$$

The classes IBQT and BO are incomparable. For finite structures, we have $\mathrm{QT}=\mathbf{B O}$.

All inclusions are clear from the definitions. Examples $S_{1}, S_{2}, S_{4}$ and $S_{5}$ given in [5] prove the strictness assertions.

\section{Axiomatizations and logically defined transformations}

The letter $B$ designates always ternary relations.

\subsection{First-order axiomatizations}

Induced betweenness in quasi-trees. Theorem 17 [5] : A structure $(N, B)$ is an induced betweenness relation in a quasi-tree (is in IBQT) if and only if it satisfies Axioms A1-A6 and A8. 


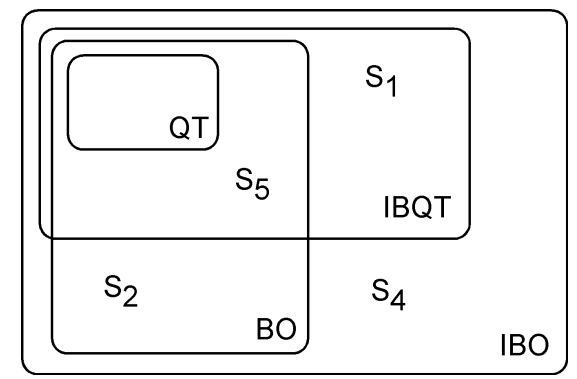

Fig. 2. Four classes and witnesses of proper inclusions.

We present a few notions for its proof. Let $S=(N, B)$ and $r \in N$. We define a binary relation on $N$ :

$$
x \leq_{r} y: \Longleftrightarrow x=y \vee y=r \vee B(x, y, r) .
$$

Lemma 18 : If $S=(N, B)$ satisfies Axioms A1-A6 and $r \in N$, then $T(S, r):=\left(N, \leq_{r}\right)$ is an O-tree.

If $S$ satisfies also A8, we will transform $T(S, r)$ into a witness that $S$ is an induced betweenness. $\square$

Lemma 19 : Let $S:=(N, B)$ satisfy A1-A6 and A8, and $r \in N$. We have $B \subseteq B_{T(S, r)}$ if $N$ is finite. $\square$

Remark and example 20 : (a) In this lemma, we may have a strict inclusion, and the inclusion $B \subseteq B_{T(S, r)}$ may be false if $S$ is infinite.

(b) The following example indicates how we can prove Theorem 17.

Let $S:=(N, B)$ such that $N:=\{0, a, b, c, d, e, f, g, h\}$ and the following conditions (and no other one) hold:

$$
\begin{aligned}
& B^{+}(0, a, b), B^{+}(0, c, d), B^{+}(0, e, f), B^{+}(0, g, h), \\
& B^{+}(b, a, c, d), B^{+}(f, e, g, h), \\
& B^{+}(b, a, 0, e, f), B^{+}(d, c, 0, e, f), B^{+}(b, a, 0, g, h), B^{+}(d, c, 0, g, h) .
\end{aligned}
$$

Figure 3(a) shows this structure without showing the last four conditions for the purpose of clarity. The curve line bacd represents $B^{+}(b, a, c, d)$.

By adding new nodes 1 and 2 to $T(S, 0)$ such that $a<1<0, c<1<0, e<$ $2<0$ and $g<2<0$, we get the rooted tree $T$ of Figure $3(b)$. Then $B=B[N]$, hence, is in IBQT. Because of the added node 1 , we have $B^{+}(b, a, c, d)$ without having $B^{+}(b, a, 0, c, d)$.

The proof of Theorem 17 consists in adding new nodes to $T(S, r)$ for such cases.

(c) If $S=(N, B)$ satisfies A1-A7 (and thus A8 by Lemma $9(2)$ ), then, for each $r \in N, T(S, r)$ is a join-tree and $B=B_{T(S, r)}$, cf. [4]. 


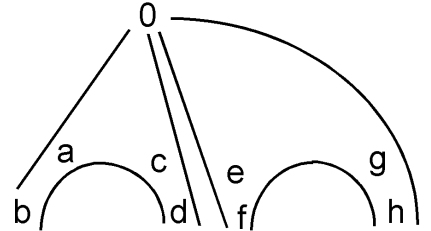

(a)

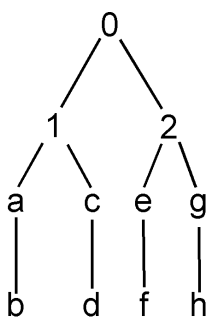

(b)

Fig. 3. ( $a$ ) shows $S$ and (b) shows $T$, Example 20.

We know from Definition 10 and Proposition 17 of [3] that a quasi-tree $(N, B)$ is the betweenness relation of a tree if and only if $B$ is discrete, i.e., that each set $[x, y]_{B}:=\{x, y\} \cup\{z \in N \mid B(x, z, y)\}$ is finite.

Corollary 21 : A nontrivial structure $(N, B)$ is an induced betweenness relation in a tree if and only if it satisfies axioms A1-A6, A8 and is discrete. These conditions are monadic second-order expressible.

Axioms A1-A6, A8 are first-order. One cannot express by a first-order sentence that a linear order $(X, \leq)$ is finite. This is expressed by the conjunction of the following conditions :

(1) $(X, \leq)$ has a minimal element $x_{0}$ and a maximal one $x_{1}$.

(2) Each $x \in X-\left\{x_{1}\right\}$ has a successor.

(3) $\left(x_{0}, x_{1}\right)$ belongs to the transitive closure of the successor relation, that exists by $(2)$.

Monadic second-order logic is necessary to express Condition (3).

Remark 22 : If $S=(N, B)$ is an induced betweenness in a quasi-tree, then any node $r$ can be taken as root for defining an O-tree $T(S, r)$ and from it, a join-tree $T$ such that $B=B_{T}[N]$. This fact generalizes the observation that the betweenness in a tree $T$ does not dependent on any root.

Informally, quasi-trees and induced betweenness in quasi-trees are "undirected notions". This will not be the case for betweenness in O-trees.

Betweenness in O-trees. We let $\mathbf{B O}_{\text {root }}$ be the class of betweenness relations of rooted $O$-trees. These relations satisfy A1-A6.

Proposition 23 : The class $\mathbf{B O}_{\text {root }}$ is axiomatized by a first-order sentence.

Proof: Consider $S=(N, B)$. If $B$ is the betweenness relation of an O-tree $(N, \leq)$ with root $r$, then, $\leq$ is nothing but $\leq_{r}$ defined before Lemma 18 from $B$ and $r$. Let $\varphi$ be the first-order (FO in short) sentence that expresses properties A1-A6 (relative to $B$ ) and the following one : 
A9 : there exists $r \in N$ such that the O-tree $T(S, r)=\left(N, \leq_{r}\right)$ whose partial order is defined by $x \leq_{r} y: \Longleftrightarrow x=y \vee y=r \vee B(x, y, r)$ has a betweenness relation $B_{T(S, r)}$ equal to $B$.

That $S$ satisfies A1-A6 insures that $\left(N, \leq_{r}\right)$ is an O-tree with root $r$. The sentence $\varphi$ holds if and only if $S$ is in $\mathbf{B O}_{\text {root }}$. When it holds, the found node $r$ defines, via $\leq_{r}$, the relevant O-tree.

An example detailed in [5] shows that $\mathbf{B O}_{\text {root }}$ is strictly included in $\mathbf{B O}$.

\subsection{Monadic second-order aximatisations}

Our second main theorem (whose proof is not straightforward) is :

Theorem 24 : The class $\mathbf{B O}$ is axiomatized by a monadic second-order sentence.

In the proof of Proposition 23, we have defined from $S=(N, B)$ satisfying A1-A6 and any node $r$ a candidate partial order $\leq_{r}$ for $\left(N, \leq_{r}\right)$ to be an O-tree with root $r$ whose betweenness relation would be $B$. The order $\leq_{r}$ being expressible by a first-order sentence, we finally obtained a first-order characterization of $\mathbf{B O}_{\text {root }}$. For $\mathbf{B O}$, a candidate order will be defined from a line, i.e., an upwards closed and linearly ordered subset, and not from a single node. (Lemmas 25 and 26 show this definition). It follows that we need for our proof, a set quantification.

Lemma 25 [Proposition 5.3 of [4]] : Let $(L, B)$ satisfy properties A1-A7' (for all $u, x, y, z \in L$, cf. Proposition 5). Let $a, b$ be distinct elements of $L$. There exists a unique linear order $\leq$ on $L$ such that $a<b$ and $B_{(L, \leq)}=B$. This order is quantifier-free definable in the logical structure $(L, B)$ in terms of $a$ and $b$. $\square$

We will denote this order by $\leq_{L, B, a, b}$. There is a quantifier-free formula $\lambda$, written with the ternary relation symbol $B$, such that, for all $a, b, u, v$ in $L$, $(L, B) \models \lambda(a, b, u, v)$ if and only if $u \leq_{L, B, a, b} v$.

A line in a structure $S=(N, B)$ that satisfies A1-A6 is a set $L \subseteq N$ of at least 3 elements in which any 3 different elements are aligned (cf. Definition $4(\mathrm{c}))$ and that is convex, i.e., $[x, y]_{B} \subseteq L$ for all $x, y$ in $L$.

Lemma 26 : Let $T=\left(N, \leq_{T}\right)$ be an O-tree, $L$ a maximal line in $T$ that has no largest node. Let $a, b \in L$, such that $a<_{L} b$, where $<_{L}$ is the restriction of $<_{T}$ to $L$.

(1) The partial order $\leq_{T}$ is first-order definable in a unique way in the structure $\left(N, B_{T}\right)$ in terms of $L, \leq_{L}, a$ and $b$.

(2) It is first-order definable in $\left(N, B_{T}\right)$ in terms of $L, a$ and $b$. 
Proof sketch of Theorem 24 : "Guess" a line $L$ in the given $S=(N, B)$ and also $a, b \in L$. An associated order $\leq$ on $N$ is FO definable from $a, b, L$ by Lemma 26. Check then that it gives an O-tree $U$ such that $B_{U}=B$. The only set quantification is for guessing the set $L$.

Next we examine in a similar perspective the class IBO. It is easy to see that $\mathbf{I B O}=\mathrm{IBO}_{\text {root }}$

Proposition 27 [5] : Every structure in the class IBO satisfies Properties A1-A6 but these properties do not characterize this class. $\square$

The construction of Theorem 24 does not extend to IBO because a finite structure in IBO may not be an induced betweenness relation of any finite Otree. No construction like the one used in the proof of Theorem 17 can produce an infinite structure from a finite one. Nevertheless :

Conjecture 28 : The class IBO is axiomatized by a monadic second-order sentence.

\subsection{Logically defined constructions}

Each betweenness relation (considered in this article) is a structure $S=(N, B)$ defined from a structure $T=\left(N^{\prime}, \leq, N\right)$ where $\left(N^{\prime}, \leq\right)$ is an O-tree and $N \subseteq N^{\prime}$, handled as a unary relation. The different cases are shown in Table 1. In each case a first-order sentence can check whether the structure $\left(N^{\prime}, \leq, N\right)$ is of the appropriate type, and the relation $B$ is first-order definable in $\left(N^{\prime}, \leq, N\right)$.

\begin{tabular}{|l|l|l|l|}
\hline $\begin{array}{c}\text { Structure } \\
(N, B)\end{array}$ & \multicolumn{1}{|c|}{ Axiomatization } & \multicolumn{1}{c|}{$\begin{array}{c}\text { Source } \\
\text { structure }\end{array}$} & \multicolumn{1}{c|}{$\begin{array}{c}\text { Trans- } \\
\text { duction }\end{array}$} \\
\hline \hline QT & FO : A1-A7, Theorem 11 & join-tree $(N, \leq, N)$ & FOT \\
\hline IBQT & FO : A1-A6, A8, Thm 17 & join-tree $\left(N^{\prime}, \leq, N\right)$ & MSOT \\
\hline BO & MSO : Theorem 24 & O-tree $(N, \leq, N)$ & MSOT \\
\hline IBO & MSO ? : Conjecture 28 & O-tree $\left(N^{\prime}, \leq, N\right)$ & not MSOT \\
\hline
\end{tabular}

Table 1

The last colomun indicates which type of logically defined transformation of structure can construct from $(N, B)$ a source structure $\left(N^{\prime}, \leq, N\right)$ witnessing its membership in the considered classes. We call transductions ${ }^{7}$ such transformations of relational structures. They are first-order transductions (FOT in short) if they are specified by FO formulas. They are monadic second-order transductions (MSOT in short) if they are specified by MSO formulas.

\footnotetext{
${ }^{7}$ By reference to Language Theory where words, terms and trees are transformed by transductions. There are strong links between language theoretical and logically defined transductions, see [6].
} 
For QT, this follows from the proof of Theorem 11 : if $S=(N, B)$ satisfies A1A7 and $r \in N$, then, the O-tree $T(S, r)=\left(N, \leq_{r}\right)$ is a join-tree and $B=B_{T(S, r)}$. For BO, the MSO sentence that axiomatizes the class constructs a relevant Otree (it guesses one, via some line, and checks that the guess is correct). For IBO, we observed that the source tree may need to be infinite for defining a finite betweenness structure, which excludes the existence of an MSO transduction, because these transformations produce structures whose domain size is linear in that of the input structure. (cf. Definition 1.6, and Chapter 7 of [6]). It remains to prove the case of IBQT. This is our third main theorem.

Theorem 29 : A join-tree $\left(N^{\prime}, \leq, N\right)$ witnessing that a given structure $S=$ $(N, B)$ is in the class IBQT can be defined from $S$ by MSO formulas.

The proof uses a notion of structuring of O-trees, adapted from the one defined in [4] for join-trees, that we will also use in Section 4. A structuring of $T$ can be seen as a set of pairwise disjoint linearly ordered subsets whose union is $\left(N_{T}, \leq_{T}\right)$.

Informally, the construction used for Theorem 17 adds to a tree some "new" nodes so as to be upper-bounds of pairs of nodes $(x, y)$. For having an MSO transduction, one can add "copies" of existing elements but not of pairs of elements. The notion of structuring makes it possible to specify a "hole" in the O-tree, i.e., a missing least upper-bound, as a copy of a single element.

We can illustrate structurings in a simple case. If in a binary tree, each node is tagged "left son" or "right son", then, a structuring consists of the set of branches (paths) starting from the root or from a right son, and going down by always going to the left son. The least upper-bound of any two nodes is then the father of some right son (above one of them). This idea is extended to O-trees, that are not join-trees in [5].

\section{Embeddings in the plane}

We give a geometric characterization of join-trees and of induced betweenness in quasi-trees, equivalently, in join-trees.

Definition 30 : Trees of lines in the plane.

(a) In the Euclidian plane, let $\mathcal{L}=\left(L_{i}\right)_{i \in \mathbb{N}}$ be a family of straight halflines (simply called lines below) with respective origins $o\left(L_{i}\right)$, that satisfies the following conditions :

(i) if $i>0$, then $o\left(L_{i}\right) \in L_{j}$ for some $j<i$,

(ii) for all $i, j \in \mathbb{N}, L_{i} \cap L_{j}$ is $\left\{o\left(L_{i}\right)\right\}$ or $\left\{o\left(L_{j}\right)\right\}$ or is empty. (We may have $\left.o\left(L_{i}\right)=o\left(L_{j}\right)\right)$.

We call $\mathcal{L}$ a tree of lines : the union of the lines $L_{i}$ is a connected set $\mathcal{L}^{\#}$ in the plane. A path (resp. a cycle) in $\mathcal{L}^{\#}$ is a homeomorphism $h$ of the interval $[0,1]$ of real numbers (respectively of the circle $S^{1}$ ) into $\mathcal{L}^{\#}$ such that $h(0)=x$ and $h(1)=y$ in the case of a path. For any two distinct $x, y \in \mathcal{L}^{\#}$, there is a 
unique path from $x$ to $y$ (it "follows the lines"), and consequently, there is no cycle. This path goes through lines $L_{k}$ such that $k \leq \max \{i, j\}$ where $x \in L_{i}$ and $y \in L_{j}$, hence, through finitely many of them. This path uses a single interval of each line it goes through, otherwise, there is a cycle.

(b) We obtain a ternary betweenness relation :

$B_{\mathcal{L}}(x, y, z): \Longleftrightarrow \neq(x, y, z)$ and $y$ is on the path between $x$ and $z$.

(c) On each line $L_{i}$, we define a linear order as follows :

$x \preceq_{i} y$ if and only if $y=x$ or $y=o\left(L_{i}\right)$ or $y$ is between $x$ and $o\left(L_{i}\right)$.

On $\mathcal{L}^{\#}$, we define a partial order by :

$x \preceq y$ if and only if $x=y$ or

$x \prec i_{i_{k}} o\left(L_{i_{k}}\right) \prec_{i_{k-1}} o\left(L_{i_{k-1}}\right) \prec_{i_{k-2}} \ldots \prec_{i_{1}} o\left(L_{i_{1}}\right) \prec_{i_{0}} y$

for some $i_{0}<i_{1}<\ldots<i_{k}$. If $k=0$, then $x \prec i_{0} y$.

It is clear that $\left(\mathcal{L}^{\#}, \preceq\right)$ is an uncountable rooted O-tree : for each $x$ in $\mathcal{L}^{\#}$, the set $\left\{y \in \mathcal{L}^{\#} \mid x \preceq y\right\}$ is linearly ordered with greatest element $o\left(L_{0}\right)$.

Definition 31 : Embeddings of join-trees in trees of lines.

Let $T=(N, \leq, \mathcal{U})$ be a structured (countable) join-tree where $\mathcal{U}$ is the set of lines. An embedding of $T$ into a tree of lines $\mathcal{L}$ is an injective mapping $m: N \rightarrow$ $\mathcal{L}^{\#}$ such that:

for each $U \in \mathcal{U}, m$ is order preserving $:(U, \leq) \rightarrow\left(L_{i}, \preceq_{i}\right)$ for some $i \in \mathbb{N}$, and

if $i \neq 0$, then $m(l s u b(U))=o\left(L_{i}\right)$.

Here, $l \operatorname{sub}(U)$ denotes the least element that is strictly above each element of $U$.

Lemma 32 : If $T$ is a structured join-tree embedded by $m$ into a tree of lines $\mathcal{L}$, then, its betweenness satisfies:

$$
B_{T}(x, y, z) \text { if and only if } \neq(x, y, z) \wedge B_{\mathcal{L}}(m(x), m(y), m(z))
$$

Theorem 33 : If $\mathcal{L}$ is a tree of lines and $N$ is a countable subset of $\mathcal{L}^{\#}$, then $S:=\left(N, B_{\mathcal{L}}[N]\right)$ is an induced betweenness in a quasi-tree. Conversely, every induced betweenness in a quasi-tree is isomorphic to $S$ as above for some tree of lines $\mathcal{L}$.

Proof : If $\mathcal{L}$ is a tree of lines and $N \subset \mathcal{L}^{\#}$ is countable, then $S:=\left(N, B_{\mathcal{L}}[N]\right)$ is in IBQT. A witnessing join-tree $T$ is built as follows. Its set of nodes is $N \cup O$ where $O$ is the set of origins of all lines in $\mathcal{L}$. Its order is the restriction to $N \cup O$ of the order $\preceq$ on $\mathcal{L}^{\#}$. Then $\left(N, B_{\mathcal{L}}[N]\right)=\left(N, B_{T}[N]\right)$ hence belongs to IBQT. 
Conversely, let $S=\left(N, B_{T}[N]\right)$ such that $T$ is a structured join-tree. It is isomorphic to $\left(N, B_{\mathcal{L}}[N]\right)$ for some tree of lines.

The construction of this tree of lines uses the fact that between two straight half-lines with same origin $A$ one can draw countably many straight half-lines with origin $A$ by choosing angles between them of the form $\alpha / 2^{i}$ for all $i$. The resulting tree of lines is clearly not printable !

\section{Concluding remarks}

We exhibit in [5] an FO class of relational structures $\mathcal{C}$ such that $\operatorname{Ind}(\mathcal{C})$, the class of induced substructures of those in $\mathcal{C}$, is not FO axiomatizable, and is even not MSO axiomatizable. This example shows that the FO characterization of IBQT does not follow by a standard logical argument from the FO characterization of the class QT, similar to the one used in Proposition 13.

Open questions : (1) We conjecture that betweenness in O-trees is not firstorder axiomatizable.

(2) We also conjecture that the class IBO of induced betweenness relations in O-trees has a monadic second-order axiomatization.

(3) In $[2,4]$, we have defined quasi-trees and join-trees of different kinds from regular infinite terms, and proved that they are equivalently the unique models of monadic second-order sentences. Both types of characterizations yield finitary descriptions and decidability results, in particular for deciding isomorphism. In a future work, we will extend to O-trees and to their betweenness relations such descriptions by regular terms, in order to get equivalences between regularity and MSO-definability.

Acknowledgement: I thank the referee for comments helping me to clarify many points.

\section{References}

1. V. Chvatal, Antimatroids, betweenness, convexity, in Research Trends in Combinatorial Optimization, Spriner, 2008, pp. 57-64.

2. B. Courcelle, Regularity equals monadic second-order definability for quasi-trees, in Fields of Logic and Computation II, Lec. Notes Comput. Sci. 9300 (2015) 129-141.

3. B. Courcelle, Several notions of rank-width for countable graphs, J. Comb. Theory, Ser. B. 123 (2017) 186-214.

4. B. Courcelle, Algebraic and logical descriptions of generalized trees, Logical Methods in Computer Science 13 (2017) Issue 3.

5. B. Courcelle, Axiomatization of betweenness in order-theoretic trees, February 2019 ,

https://hal.archives-ouvertes.fr/hal-02205829

6. B. Courcelle and J. Engelfriet, Graph structure and monadic second-order logic, a language theoretic approach, Cambridge University Press, 2012. 
7. J. Lihova, Strict-order betweenness, Acta Univ. M. Belii Ser. Math. 8 (2000) 27-33.

8. S. Oum, Rank-width and vertex-minors. J. Comb. Theory, Ser. B 95 (2005) 79100. 